\title{
Tumors induce coordinate growth of artery, vein, and lymphatic vessel triads
}

\author{
Alanna Ruddell ${ }^{1,2^{*}}$, Alexandra Croft ${ }^{2}$, Karen Kelly-Spratt ${ }^{1}$, Momoko Furuya ${ }^{1}$ and Christopher J Kemp ${ }^{1,3}$
}

\begin{abstract}
Background: Tumors drive blood vessel growth to obtain oxygen and nutrients to support tumor expansion, and they also can induce lymphatic vessel growth to facilitate fluid drainage and metastasis. These processes have generally been studied separately, so that it is not known how peritumoral blood and lymphatic vessels grow relative to each other.
\end{abstract}

Methods: The murine B16-F10 melanoma and chemically-induced squamous cell carcinoma models were employed to analyze large red-colored vessels growing between flank tumors and draining lymph nodes. Immunostaining and microscopy in combination with dye injection studies were used to characterize these vessels.

Results: Each peritumoral red-colored vessel was found to consist of a triad of collecting lymphatic vessel, vein, and artery, that were all enlarged. Peritumoral veins and arteries were both functional, as detected by intravenous dye injection. The enlarged lymphatic vessels were functional in most mice by subcutaneous dye injection assay, however tumor growth sometimes blocked lymph drainage to regional lymph nodes. Large red-colored vessels also grew between benign papillomas or invasive squamous cell carcinomas and regional lymph nodes in chemical carcinogen-treated mice. Immunostaining of the red-colored vessels again identified the clustered growth of enlarged collecting lymphatics, veins, and arteries in the vicinity of these spontaneously arising tumors.

Conclusions: Implanted and spontaneously arising tumors induce coordinate growth of blood and lymphatic vessel triads. Many of these vessel triads are enlarged over several $\mathrm{cm}$ distance between the tumor and regional lymph nodes. Lymphatic drainage was sometimes blocked in mice before lymph node metastasis was detected, suggesting that an unknown mechanism alters lymph drainage patterns before tumors reach draining lymph nodes.

Keywords: Melanoma, Carcinoma, Lymphangiogenesis, Lymphatic vessel, Angiogenesis, Blood vessel

\section{Background}

Blood and lymphatic vessels arise during embryogenesis and mature by an orderly program of gene expression, signaling, and morphogenesis that establishes a triad arrangement of lymphatic, vein, and artery throughout the body $[1,2]$. The formation of each vessel type is regulated by different combinations of transcription factors (e.g. Notch, COUP-TFII, and Prox1), While some factors specifically regulate lymphatic (Prox1), vein (COUP-TFII), or artery formation (Hey1/2), other vascular signaling pathways show overlapping activities (e.g. Sox transcription factors, and vascular endothelial growth factors (VEGFs)

\footnotetext{
* Correspondence: ruddella@uw.edu

'Fred Hutchinson Cancer Research Center, Seattle, WA, USA

${ }^{2}$ Department of Comparative Medicine, University of Washington School of Medicine, 1959 NE Pacific St., Box 357190, Seattle, WA 98195, USA
}

Full list of author information is available at the end of the article
[3-5]. Intercellular signaling of proteins including semaphorins, ephrins, and chemokines helps to pattern formation of the network of lymphatic and blood vessels, while VEGF family members are important to support growth of the lymphatic and blood vasculature. Mature triads of lymphatics, arteries, and veins are maintained in the adult unless activated by events requiring vascularization such as tumorigenesis [6].

Tumors induce extensive growth of blood vessels to obtain oxygen and nutrients essential for their continued expansion and progression $[7,8]$. The resulting blood vessels are variable and abnormal, ranging in size and morphology from capillaries and small blood vessels to larger feeder arteries and draining veins surrounding the tumor vascular bed [9]. The lymphatic vasculature can also expand [10-12], to increase lymph drainage from 
tumors featuring high interstitial fluid pressure [13,14], and also to promote metastasis of tumors to draining lymph nodes (LNs) [15,16]. Tumors can produce growth factors promoting angiogenesis (eg. VEGF-A, placental growth factor), as well as lymphangiogenesis (VEGF-C, -D), although the resulting vessels are highly abnormal relative to those of embryos $[17,18]$, presumably due to aberrant signaling $[9,19]$. These vessels can actively increase blood circulation and lymph drainage, or they can become so abnormal that they are unable to transport blood [20] or lymph $[14,21]$.

Tumor-induced angiogenesis and lymphangiogenesis are generally studied separately, raising the question of whether the normal triad pattern of lymphatic, vein, and artery is used to support tumor vessel growth, or if blood and lymphatic vessels are independently generated and patterned during tumor growth. In this study we utilized two murine models to compare the growth of large peritumoral lymphatic and blood vessels. The syngeneic B16-F10 melanoma model [22] permits ready visualization of peritumoral vasculature on the flank skin of $\mathrm{C} 57 \mathrm{Bl} / 6$ mice, and these tumors metastasize via the lymphatics to draining lymph nodes (LNs) [23-25]. The second model used was an autochthonous squamous cell carcinoma induced by DMBA mutagenesis, followed by repeated TPA promoter treatments of outbred mice [26]. These naturally arising tumors first present as exophytic benign papillomas, which can convert to endophytic invasive carcinomas capable of metastasizing to draining LNs or to the lung. Both of these tumor models induced growth of large red-colored blood vessels in the skin extending several $\mathrm{cm}$ between the primary tumor and regional draining LNs. Immunohistochemical and functional analysis determined that these enlarged vessels included large arteries, veins, and one or more lymphatics, indicating that tumor-induced vessel growth utilizes the same patterning of vessel triads established during development.

\section{Methods}

\section{Mouse tumor models}

Mice were maintained in micro-isolator rooms under specific pathogen-free conditions. Experiments involving animals were approved by the FHCRC Animal Care and Use Committee. For the B16-F10 tumor model, four week-old $\mathrm{C} 57 \mathrm{Bl} / 6$ mice were purchased from Jackson Laboratories (Bar Harbor, ME). B16-F10 murine melanoma cells [27] from the American Type Culture Collection (Manassas, VA) were tested to confirm that the cells were free of mycoplasma contamination (Cambrex, Rockland, $\mathrm{MD})$. Mice were anesthetized with isoflurane and subcutaneously injected in the left flank with 15,000 B16-F10 cells in $50 \mu \mathrm{l}$ of Hanks' buffered saline solution, while the control right flanks were injected with $50 \mu \mathrm{l}$ of Hanks' buffered saline solution [24,25]. Mice were euthanized after 14-23 days, when tumors were $1-2 \mathrm{~cm}$ in diameter. Vessel diameter was measured using a ruler. Using a scalpel, single large red-colored blood vessels in the flank skin were dissected in $2 \mathrm{~mm} \times 5$ sections. Tissue was fixed in $4 \%$ paraformaldehyde at room temp for $4 \mathrm{~h}$, incubated in $30 \%$ sucrose/PBS overnight at $4^{\circ} \mathrm{C}$, and mounted in Tissue-Tek O.C.T. compound (Sakura; Torrance, CA) before cryosectioning at $-25^{\circ} \mathrm{C}$.

For the DMBA/TPA squamous cell carcinoma model, wild-type $\mathrm{NIH} / \mathrm{Ola}$ or $\mathrm{p} 19^{\mathrm{Arf+/}}$ mice were treated on the shaved dorsal skin with DMBA, and then twice weekly with TPA for 15 weeks to induce papillomas and squamous cell carcinomas, as previously described $[28,29]$. Mice were euthanized 28 to 32 weeks after DMBA treatment, or if moribund. More than a dozen papillomas or carcinomas arose on the back skin of each mouse, with diameters ranging from 2 to $15 \mathrm{~mm}$. Large vessels draining papillomas or carcinomas were dissected from the interior surface of the skin in $2 \mathrm{~mm} \times 5 \mathrm{~mm}$ sections with a scalpel, and mounted in O.C.T. for cryosectioning.

\section{Functional labeling of lymphatic and blood vessels}

Functional lymphatic vessels were identified by lymphography, injecting isoflurane-anesthetized mice subcutaneously in the base of the tail with $0.25 \%$ lysine-fixable Texas Red Dextran (Invitrogen; Gran Island, NY) and 2.5\% Evans Blue (Sigma; St. Louis, MO) in $100 \mu$ l Hanks' buffered saline solution, followed $20 \mathrm{~min}$ later by $\mathrm{CO}_{2}$ overdose and necropsy. The blood circulation was labeled by injecting mice intravenously in the tail vein with $0.05 \%$ Fluorescein isothiocyanate (FITC) - Lycopersicon esculentum lectin (Vector Laboratories; Burlingame, CA) in $100 \mu \mathrm{l}$ Hank's buffered saline solution in the tail vein, immediately followed by $\mathrm{CO}_{2}$ overdose, followed by dissection of vessels. Vessels were mounted in OCT for cryosectioning.

\section{Immunostaining analysis and vessel quantitation}

Twelve $\mu \mathrm{m}$ cryosections were fixed in $4 \%$ paraformaldehyde for $10 \mathrm{~min}$, followed by incubation in $0.3 \%$ hydrogen peroxide in methanol for $30 \mathrm{~min}$. Sections were then immunostained with CD31 (BD Pharmingen), MECA -32 (BD Pharmingen), LYVE-1 (Ebioscience), or 10.1.1 [30], as previously described [24]. Immunohistochemical staining was detected using horseradish peroxidase-labeled secondary antibodies and Vector VIP detection, followed by brief methyl green counterstaining at room temperature (Vector Laboratories). In some experiments, sections were immunostained with 10.1.1 and Prox1 (Millipore; Billerica, MD), and detected with Alexa 568-anti-hamster and Alexa 488-conjugated anti-rabbit antibodies (Invitrogen; Sparks, MD), respectively, followed by mounting in Prolong Gold (Invitrogen). 


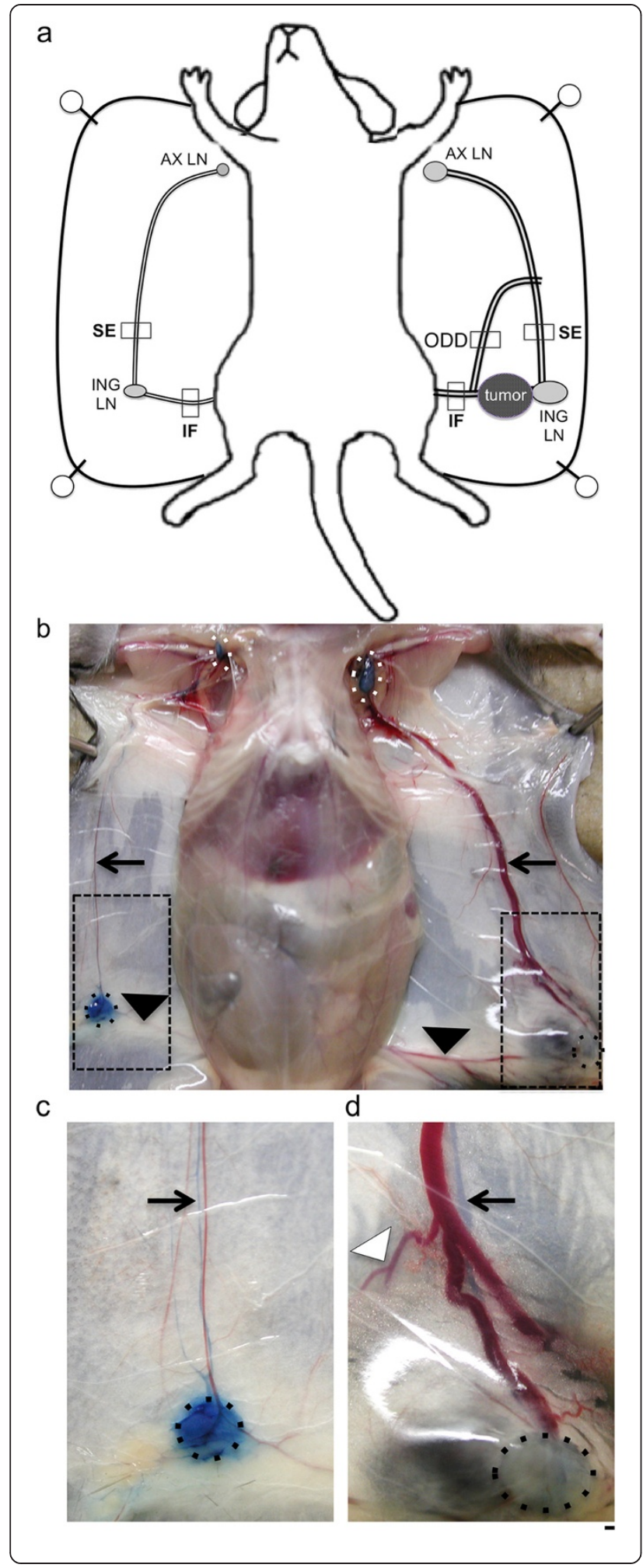

Figure 1 Growth of peritumoral lymphatic and blood vessels in a melanoma model. a). Schematic of B16-F10 melanoma flank tumor, associated SE, IF, and ODD vessels, and regional LNs. The boxes around vessels illustrate regions dissected for microscopic analysis. b). Necropsy of melanoma-bearing mouse identifies greatly enlarged red-colored peritumoral vessels. The tumor-draining AX LNs are outlined by dotted white circles, while dotted black circles outline the ING LNs. The arrows point to the SE vessels, while the black arrowheads identify the IF vessels. The dotted black boxes show the flank regions analyzed at higher magnification in (c) and (d). c) Arrow points to Evans Blue-filled SE lymphatic vessel adjacent to red-colored SE blood vessel on normal flank. d). Arrow points to enlarged Evans Blue-filled SE lymphatic vessel draining tumor and ING LN, next to enlarged red-colored blood vessels. The white arrowhead identifies an ODD vessel not seen in the normal flank skin. Scale bar $1 \mathrm{~mm}$.

For measurements of 10.1.1- or CD31-positive lymphatic, vein, and artery size, three sections from each vessel were viewed at 100x magnification, and vessels were outlined to measure the area using the NIS Elements BR 3.0 program (Nikon, Inc.; Melville, NY). Area measurements were analyzed for significance by Mann-Whitney $\mathrm{U}$ two-tailed test.

\section{Results}

Peritumoral blood and lymphatic vessels are enlarged

Prominent red-colored blood vessels surround B16-F10 melanoma tumors implanted in the flank of syngeneic $\mathrm{C} 57 \mathrm{Bl} / 6$ wild-type mice (Figure 1a), often growing toward regional draining LNs. These enlarged vessels (Figure 1b) often reach several millimeters in diameter (Figure 1d) as compared to the normal small vessels of the contralateral non-tumor-draining flank (Figure 1c). The affected vasculature in the flank region included the major superficial epigastric blood vessel (SE) connecting the inguinal (ING) and axillary (AX) LNs along the milk line, and the inguinofemoral (IF) vessel connecting the inguinal LN with central vessels (Figure 1a). Other abnormal red-colored vessels (ODD) variably appeared in adjacent regions of the flank skin (e.g. arrowhead, Figure 1d) in regions that normally do not contain large blood vessels (Figure 1c). These phenotypes of increased SE, IF, and ODD vessel growth were identified in all twelve of the tumor-bearing mice analyzed in this study.

We tested whether this angiogenesis also involves lymphatic vessels, as the SE blood vessel between the ING and AX LNs tracks with a major lymphatic vessel draining from the ING LN [31]. A functional assay was used to identify lymphatic vessels associated with the tumor blood vessels, by injecting Evans Blue dye subcutaneously into the base of the tail to label the ING and AX LN lymphatic drainage on both flanks [32]. This injection identified the blue collecting lymphatic vessel adjacent to the SE blood vessel (Figure 1b). The labeled SE lymphatic vessel was much larger on the tumor- 
draining flank (Figure 1d) relative to the contralateral control flank (Figure 1c). These tumors therefore induced enlargement of the SE lymphatic vessel adjacent to the SE blood vessels. The ING and AX LNs on each side also labeled with Evans Blue (Figure 1b), indicating that this lymphatic drainage is not blocked by tumor growth. The second-tier AX LN preferentially filled with Evans Blue dye on the tumor-draining side relative to the control side (Figure 1b), consistent with our previous reports that B16-F10 tumors accelerate lymph drainage $[24,33]$.

\section{Identification of functional lymphatic and blood vessels}

The composition of the SE vessels was investigated by immunostaining with antibodies to lymphatic and blood endothelial surface markers. Fixation of the dissected vessel in paraformaldehyde followed by $30 \%$ sucrose impregnation was used to preserve the red blood cells within blood vessels to confirm their identify. This fixation technique also helped to keep the vessels patent to facilitate comparison of their size. First, a normal SE vessel was dissected, cryosectioned, and immunostained. The CD31 antibody recognizes PECAM expressed on lymphatics, veins, and arteries [34]. CD31 immunostaining revealed that the single SE "vessel" consisted of a grouped lymphatic, vein, and artery in normal mice (Figure 2a). The 10.1.1 antibody recognizes mCLCA1
[35], which is expressed on the surface of lymphatic but not blood endothelium [36]. 10.1.1 antibody immunostaining identified the collecting lymphatic vessel but not the SE blood vessels (Figure 2b). The SE lymphatic vessel was negative for LYVE-1 (data not shown), consistent with previous reports that collecting lymphatics do not express LYVE-1 [37]. The vascular endothelial marker antibody MECA-32 variably immunostained these vessels under the fixation conditions used (data not shown), and is reported to be variably expressed in tumor blood vessels [38]. The 10.1.1 and CD31 antibodies were therefore selected as the best combination to distinguish lymphatic and blood vessels by immunostaining. The vessels are further distinguished from each other by their anatomical characteristics, as arteries are rounded with a narrow lumen and thick wall, while veins and lymphatics are thin-walled with a larger irregularly shaped lumen.

Dye injection assays were used to functionally distinguish these vessels. Intravenous injection of FITC lysopersicon esculentum lectin [20] labeled the vascular endothelium and erythrocytes of the SE artery and vein, but not the lymphatic vessel of normal control mice (Figure 2c). Subcutaneous injection of lysine-fixable Texas Red Dextran (15 kd MW) into the lateral tail base [32] preferentially labeled the draining lymphatic vessel (Figure 2d). These functional immunostaining assays
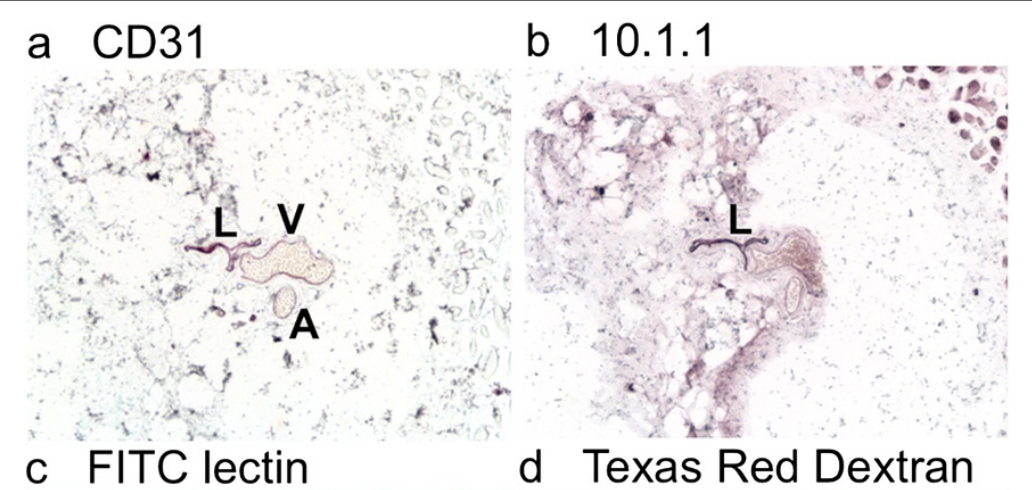

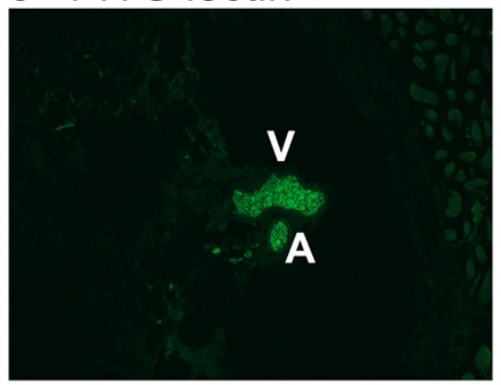

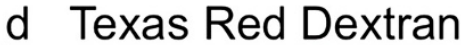

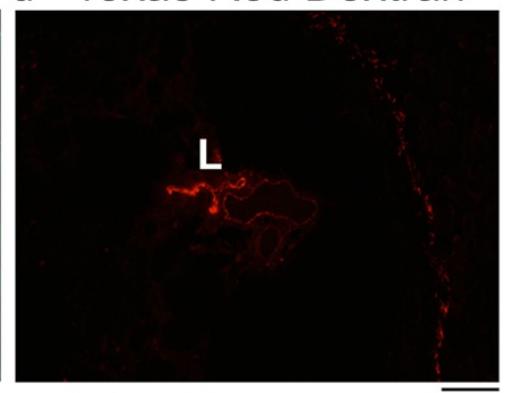

Figure 2 Triad arrangement of normal SE lymphatic, vein, and artery. a). CD31 immunohistochemical purple staining of lymphatic ( $L$ ) vein $(\mathrm{V})$ and artery (a), with Methyl Green nuclear counterstaining. b). 10.1.1 immunohistochemical staining identifies the SE lymphatic vessel. The nonspecific staining in the upper right corner is myoglobin pseudoperoxidase-positive muscle. c). Intravenously injected FITC L. Iycopersicum lectin labels the artery and vein, demonstrating that they are functional in circulating blood. d). Texas Red Dextran injected subcutaneously into the tail base labels the lymphatic vessel, indicating that it is functional in circulating lymph. Scale bar $50 \mu \mathrm{m}$. 
demonstrate that intravenously injected FITC lectin labels CD31-positive blood vessels, while subcutaneouslydelivered Texas Red Dextran identifies 10.1.1-positive lymphatic vessels.

\section{Peritumoral growth of lymphatic, artery, and vein triads} The enlarged SE vessels draining B16-F10 tumors were characterized by cryosectioning and immunostaining with the 10.1.1 and CD31 antibodies, and by comparison with the morphology of normal contralateral SE vessels, which consist of an artery, vein, and one or more lymphatic vessels. CD31 immunostaining identified a large tumordraining vein, artery, and lymphatic vessel (Figure 3a), while 10.1.1 immunostaining identified only the lymphatic vessel (Figure 3b). These peritumoral blood and lymphatic vessels are much larger than the corresponding normal contralateral vessels (Figure 2), in agreement with the grossly visible enlargement of the red-colored blood vessels and Evans Blue dye-positive lymphatic vessels of tumor-bearing mice (Figure 1b). This enlargement of SE lymphatic and blood vessels was a consistent feature of B16-F10 tumors growing on the flank. This growth effect spread over distances up to several centimeters away from the tumor, as enlargement of the SE lymphatic and blood vessels extends all the way to the AX LN (Figure 1b).
Dye was injected subcutaneously into the tail to test whether the enlarged tumor-draining lymphatic vessels are able to transport fluid, as tumor-draining lymphatics can be compressed and nonfunctional [21]. Injected Texas Red Dextran labeled the 10.1.1 Abpositive lymphatic vessel, indicating that it is functional in draining lymph from the tail past the tumor and through the ING LN to travel through the efferent SE lymphatic vessels (Figure 3c). In addition, the artery and vein were labeled with Texas Red Dextran (Figure 3c), indicating that the dye traveled from the lymphatic drainage to the heart and into the blood circulation 20 minutes after injection. [33]. This is consistent with our previous reports that dye reaches the vascular circulation within minutes in mice with cancer, due to increased lymph flow [33,36]. The circulation of subcutaneously injected lectin in lymphatic as well as blood vessels reveals that both types of vessels are functional.

Prox1 immunostaining was used to confirm the lymphatic identity of the abnormally enlarged 10.1.1 Ab-positive SE lymphatic vessel, as Prox1 is a transcription factor expressed in lymphatic but not vascular endothelium [39]. The endothelial cells of the SE lymphatic vessel of Figure $3 \mathrm{c}$ and adjacent lymphatic vessels co-expressed surface 10.1.1 (Figure 3d) and nuclear Prox1 (Figure 3e, f),

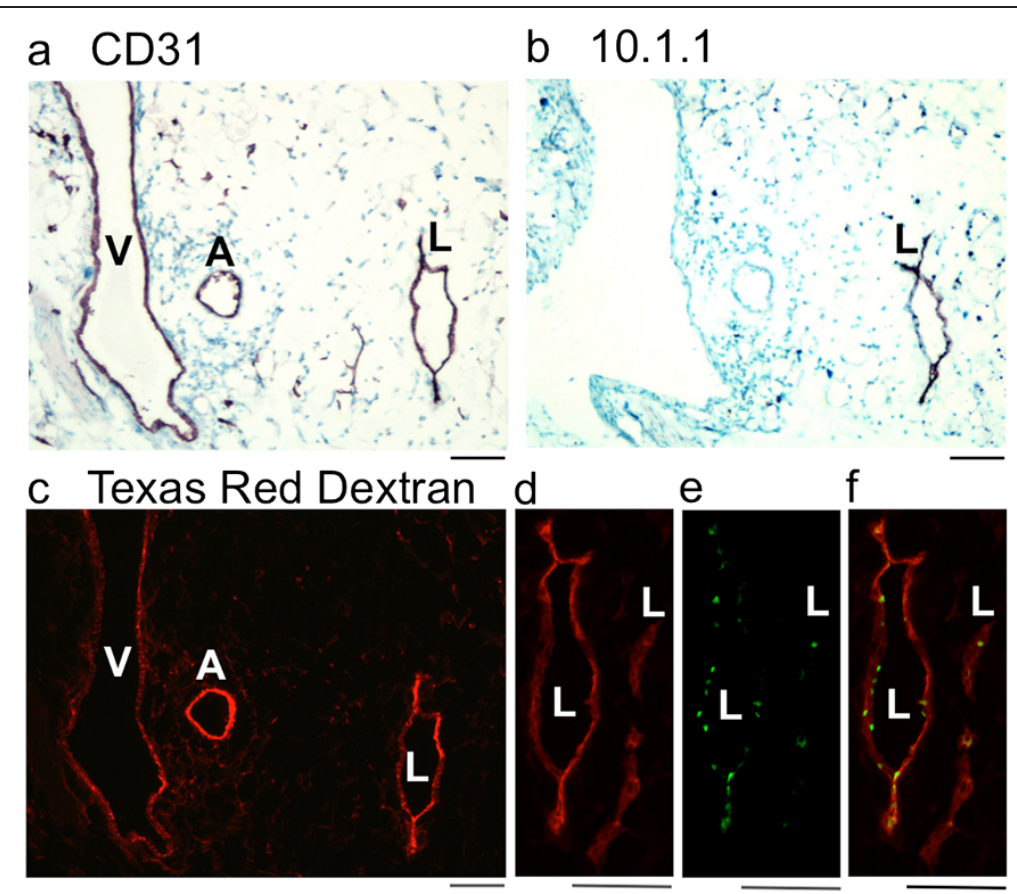

Figure 3 Enlarged and functional peritumoral SE lymphatic, vein, and artery triad. a). CD31 immunohistochemical staining identifies enlarged and abnormal triad of peritumoral SE lymphatic, vein, and artery. b). 10.1.1 immunohistochemical staining of the same vessel triad identifies the SE lymphatic vessel. c). Subcutaneously injected Texas Red Dextran circulates through the peritumoral SE lymphatic vessel and then into the SE vein and artery after $20 \mathrm{~min}$. d). Red 10.1.1 immunofluorescent staining of an adjacent section identifies the lymphatic vessel shown in (c), and an additional collapsed lymphatic vessel. e). Green nuclear Prox1 immunofluorescent staining identifies the endothelium of both lymphatic vessels. f). Merging of the 10.1.1 (d) and Prox1 images (e) confirms that the 10.1.1-positive lymphatic vessels express Prox1. Scale bars $50 \mu \mathrm{m}$. 
confirming that these abnormal vessels are lymphatics. These findings identify the 10.1.1 antibody as a marker of collecting lymphatic vessels, in addition to its previously described utility for identification of initial lymphatic vessels [36].

The visible enlargement of SE and IF lymphatic and blood vessels was consistently observed in the 12 melanomabearing mice used for these studies. The growth of the SE lymphatic, vein, and artery was compared by estimating the area of each vessel from immunostaining images. The estimated area of the tumor-draining lymphatics, veins and arteries increased 12-, 9-, and 6-fold relative to uninvolved contralateral SE vessels, respectively (Figure 4), and these increases were all statistically significant by $\mathrm{t}$ test $(\mathrm{p}<0.0001)$. These increases are likely underestimates, as the vessels were often collapsed after dissection (eg. lymphatic vessel in Figure 3b). Nonetheless, these comparisons confirm the visual observations that B16-F10 melanomas consistently induce growth of enlarged triads including lymphatics, veins, and artery.

\section{Coordinate growth of peritumoral lymphatic and blood vessels}

B16-F10 tumor growth in the flank was associated with other enlarged vessels in addition to the SE vessels (Figure 1b), which could potentially also involve clustered growth of lymphatic, vein, and artery. The inguinofemoral (IF) blood vessel connecting the ING LN with the midline was consistently enlarged in the area of the tumor and where it travels dorsal to the ING LN to join the SE vessels (black arrowhead, Figure 1b). Variable "new" vessels were also seen near the tumor, and also appearing at some distance branching from the SE or IF vessels (e.g. white arrowhead, Figure 1d). These

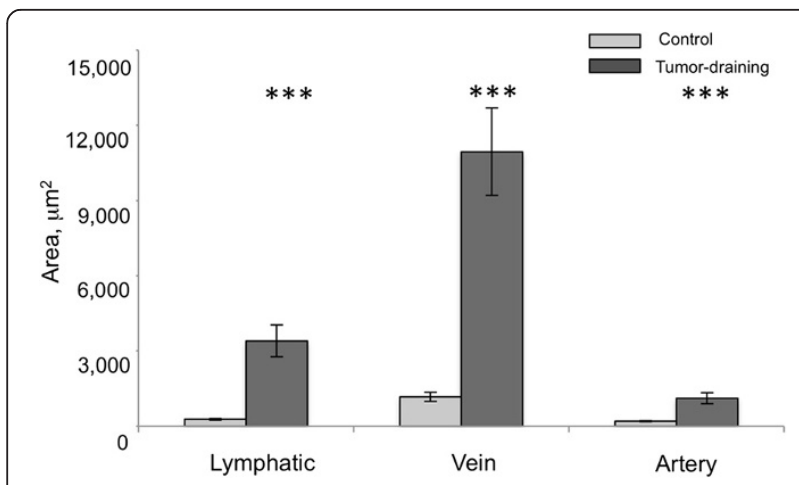

Figure 4 Coordinate growth of lymphatics, veins, and arteries in triads. Area of tumor-draining and contralateral control SE lymphatics, veins, and arteries were estimated by quantitation of immunostained images. Data is averaged from 18 measurements of vessel triads from 6 mice. The tumor-draining lymphatics, veins and arteries are all significantly enlarged (Mann Whitney $U$ two-tailed test $\left.^{*}{ }^{* *}, \mathrm{p}<0.0001\right)$. vessels were dissected, cryosectioned, and analyzed by immunostaining. CD31 immunostaining of the contralateral non-tumor-draining IF vessels identified a normal small lymphatic, vein, and artery close to each other (Figure 5a), while 10.1.1 Ab immunostaining was restricted to the lymphatic vessel (Figure 5b). Subcutaneous Texas Red Dextran injection identified all three of the vessels (Figure 5c), confirming that that these vessels are all functional, and that increased lymphatic drainage in this tumor-bearing mouse rapidly delivers lymph into the blood circulation.

The IF vessel dissected from the tumor-bearing flank also included a lymphatic, vein, and artery (Figure 5d, e), that were all enlarged relative to the contralateral control vessels (Figure 5a, b). Subcutaneously delivered Texas Red Dextran identified the vein and artery but not the lymphatic vessel (Figure 5f). Tumor growth in this particular mouse therefore blocked IF lymphatic drainage, while other tail-draining lymphatic vessels instead delivered Texas Red Dextran to the blood circulation.

The ODD vessels also consisted of triads of enlarged lymphatics, veins, and arteries by immunostaining for CD31 (Figure 5g) and 10.1.1 (Figure 5h). In the example shown, the lymphatic and blood vessels were all functional, as subcutaneously injected Texas Red Dextran labeled lymphatic as well as blood vessels (Figure 5i). There are no vessels visible in comparable regions of the contralateral control flank (Figure 1c) so that it remains to be determined whether these ODD vessels are derived by expansion of pre-existing very small vessel triads, or by some other mechanism.

\section{Pre-metastatic tumors sometimes block lymph drainage}

Lymph drainage was occasionally impaired by growth of B16-F10 tumors, as detected by failure of subcutaneously injected Texas Red Dextran to appear in the tumordraining lymphatic vessel, while it reached the circulation via other lymphatics to be detected in the blood vessels (e.g. Figure 5f). This phenotype was further investigated by examining the visible drainage of Evans Blue Dye into the ING and AX LNs. Subcutaneous Evans Blue injection into the tail generally labeled both the ING and AX LNs (dotted circles, Figure 1b) in 8 of 12 tumor-bearing mice injected with dye. However, 4 of the 12 mice showed blocked drainage on the tumor-draining side, with blue dye draining to the ING LN (Figure 6e) but not continuing to the AX LN on the tumor-draining side (Figure $6 \mathrm{c}$ ), while dye drainage through the control contralateral ING (Figure 6d) and AX LN (Figure 6b) was unimpeded. The ING or AX LNs of these mice did not contain visible melanotic metastases, even though melanotic micrometastases are readily visible on the LN cortex [23] indicating that the tumors likely block lymph drainage by some other mechanism. 


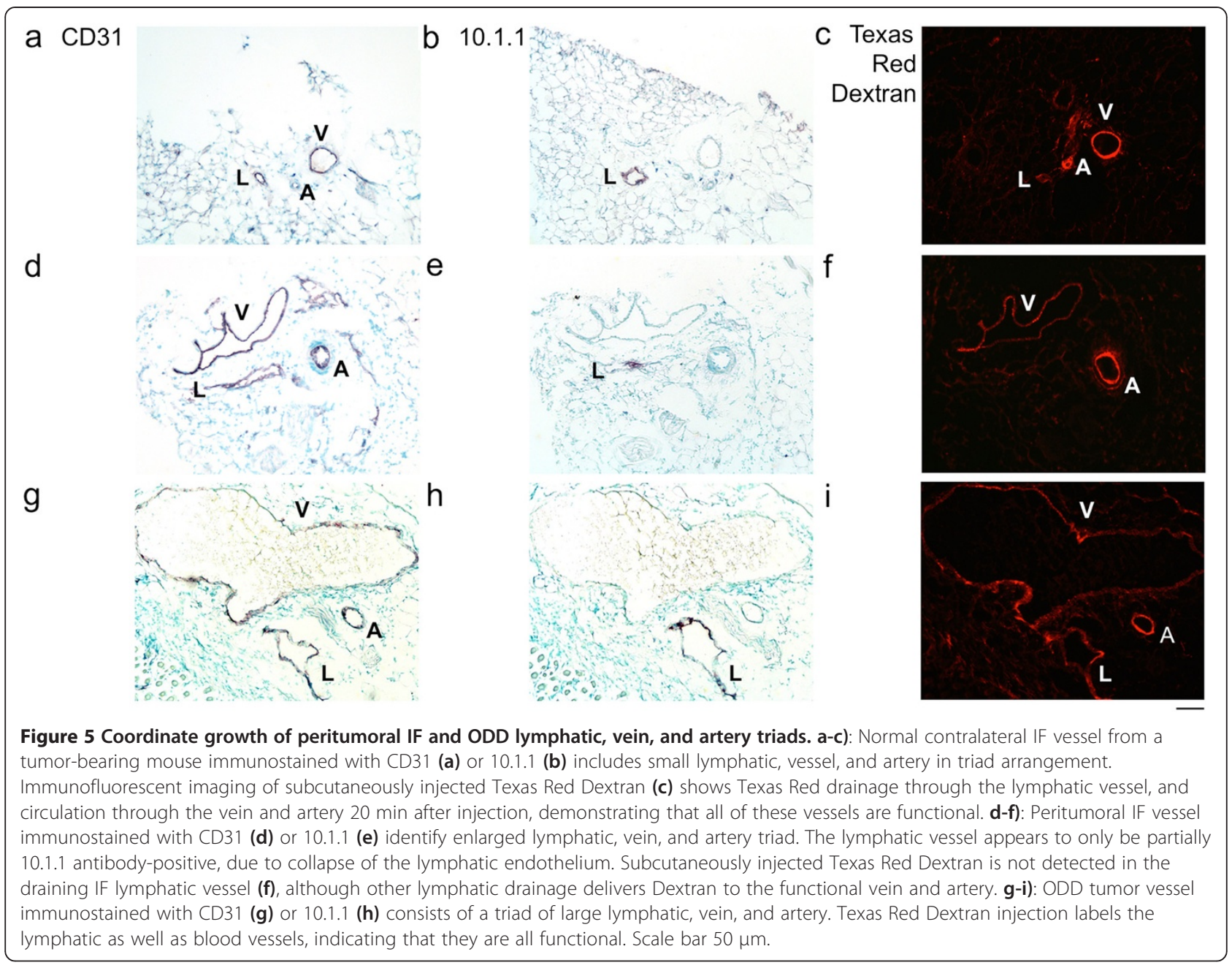

Coordinate growth of lymphatic, vein, and artery triads in an autochthonous tumor model

A murine squamous cell carcinoma model was used to test whether blood and lymphatic vessel triads are also a feature of more slowly growing spontaneous tumors. DMBA mutagenesis followed by TPA promoter treatments in outbred mice $[26,29]$ results in the appearance of benign papillomas on the treated dorsal skin after 3 to 9 months (Figure 7b), a subset of which sometimes convert to invasive carcinomas (Figure 7c). Tumor growth and malignant progression is accelerated in mice carrying a germline deletion in the p19/Arf tumor suppressor gene [28]. Inspection of the ventral surface of the dorsal skin of tumor-bearing wild-type or $\mathrm{p} 19^{\mathrm{Arf+} /-}$ mice during necropsy identified abnormal and enlarged blood vessels draining papillomas (Figure 7e) and carcinomas (Figure 7f) relative to normal dorsal skin that featured only a few tiny blood vessels (Figure $7 \mathrm{~d}$ ). The tumor-draining blood vessels often extended to the draining AX LNs [28], resembling the growth of peritumoral B16-F10 blood vessels toward the ING or AX LNs (Figure 1a).
Vessels draining benign papillomas from 3 mice were dissected, sectioned and immunostained, to identify triads including thick-walled arteries and veins (Figure $7 \mathrm{~g}$ ), as detected by immunostaining for vascular endothelial marker MECA-32 [40]. Multiple lymphatics were detected by 10.1.1 immunostaining (Figure 7h). These slowly growing benign tumors thus also induce coordinate growth of large vessel triads, with the arteries particularly enlarged relative to the veins and lymphatics. There are no major vessels in the dorsal skin of mice (Figure $7 \mathrm{~d}$ ), so that the origin of these peritumoral vessels is not obvious.

Immunostaining analysis of the red-colored vessels surrounding DMBA/TPA-induced carcinomas from 6 mice also identified triads of vein, artery, and lymphatics by CD31 (Figure 7i) and 10.1.1 (Figure 7j) immunostaining. These vessels were greatly enlarged and abnormal, resembling the vessels draining B16-F10 melanoma tumors (Figure 3a). The lymphatic vessel shown in Figure $7 \mathrm{j}$ included 10.1.1 Ab-positive flaps that likely are valves typical of collecting lymphatic vessels [41,42], 


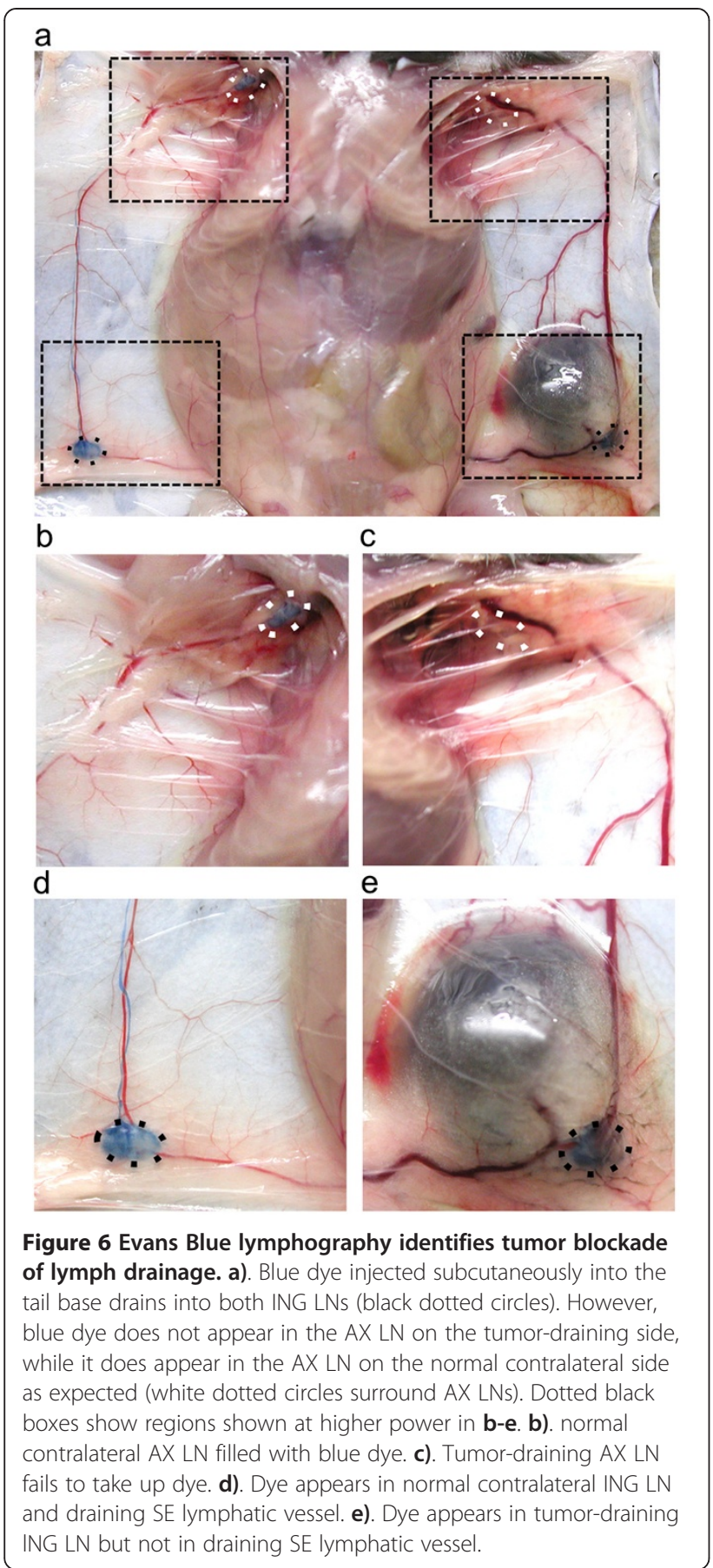

which were a common feature of these peritumoral lymphatics (data not shown). Other carcinoma-associated vessels showed more extreme enlargement and disorganization, although they maintained clustering of multiple blood vessels (Figure 7k) and lymphatics (Figure 7l). These findings identify coordinate growth of peritumoral lymphatic and blood vessels in autochthonous papillomas and carcinomas.
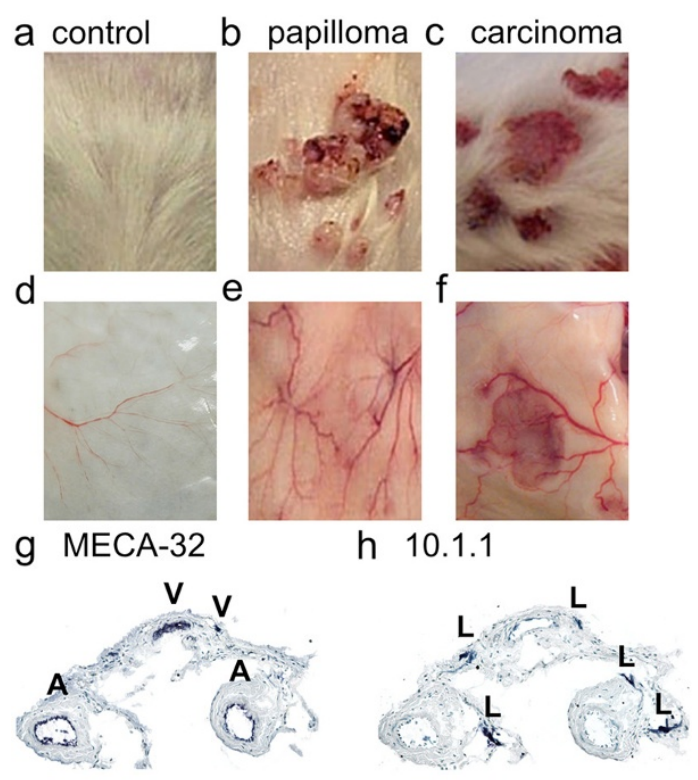

i CD31
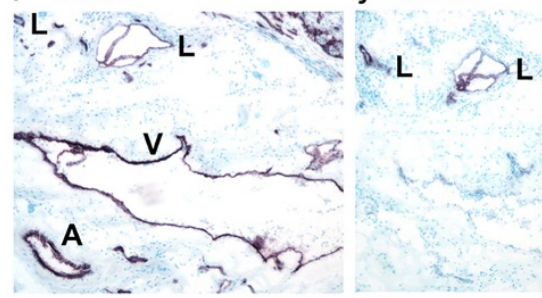

k CD31

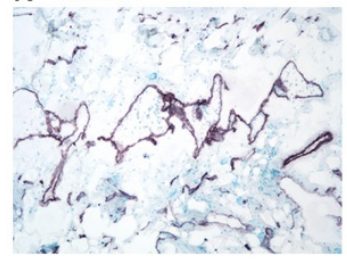

| 10.1 .1

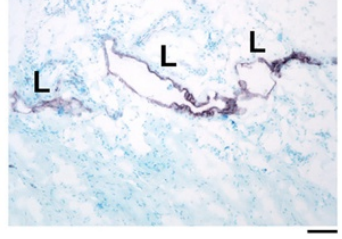

Figure 7 Large vessel triads containing artery, vein, and lymphatics arise in autochthonous tumors. a). The dorsal skin of a control mouse. b). The dorsal skin of a p19 $9^{\text {Arf }+/-}$ mouse bearing DMBA/TPA- induced papillomas. c). The dorsal skin of a p19 Arf+/mouse bearing DMBA/TPA-induced invasive carcinomas. d). Normal ventral skin is relatively avascular with sparse and very small blood vessels. e). Ventral skin beneath the papillomas (b) shows highly abnormal and enlarged red-colored vessels. f). The ventral skin beneath the carcinomas (c) shows highly abnormal and enlarged red-colored vessels. g). MECA-32 immunohistochemical staining of a papilloma-associated vessel from a p19 $9^{\text {Arft/- }}$ mouse identifies large veins and thick walled arteries. h). 10.1.1 immunohistochemical staining of the same papilloma-associated vessel (g) identifies five lymphatic vessels. i). CD31 immunohistochemical staining of a carcinoma-associated vessel from a wild-type mouse identifies large lymphatics, vein, and artery. j). 10.1.1 immunohistochemical staining of the same carcinoma-associated vessel (i) identifies 2 lymphatic vessels. k). CD31 immunohistochemical staining of a carcinoma-associated vessel from another wild-type mouse identifies disorganized cluster of enlarged blood and lymphatic vessels. I). 10.1.1 immunohistochemical staining of the same carcinoma-associated vessel (k) identifies clustered enlarged lymphatic vessels. Scale bar $50 \mu \mathrm{m}$. 


\section{Discussion and conclusions}

The enlarged red-colored vessels arising over some distance from spontaneous or implanted tumors include clustered lymphatics, veins, and arteries, demonstrating that the triad arrangement typical of large vessels is utilized during tumor-induced angiogenesis and lymphangiogenesis. The peritumoral SE and IF vessels likely grow by enlargement of the pre-existing normal vessel triads. Blood and lymphatic vessels can both enlarge by endothelial proliferation, incorporation of bone marrowderived cells $[43,44]$, and/or by thinning and dilation [45]. The "odd" vessels that appear surrounding melanomas, papillomas, and carcinomas could similarly be generated by expansion from very small vessel triads, or they could potentially arise by recapitulation of their initial formation in embryos $[3,46,47]$. The latter possibility could involve assembly of endothelial progenitors, as occurs in development of the dorsal artery and cardinal vein $[48,49]$, or sprouting of lymphatic vessels from small veins [50,51]. Further studies of the generation of these "odd" vessel triads growing around tumors will be required to identify the mechanisms guiding this coordinate blood and lymphatic vessel growth.

Many of the peritumoral vessels grow toward the regional LNs in the melanoma and squamous cell carcinoma models, raising the question of how this expansion is related to lymph node metastasis. Only the lymphatic vessel would directly connect the tumor to the draining $\mathrm{LN}$ to deliver tumor cells, so that the enlargement of functional lymphatic vessels could facilitate LN metastasis. In fact, prostaglandin and VEGF-D-induced enlargement of collecting lymphatic vessels has recently been demonstrated to promote metastasis to draining LNs [45]. Other studies have found that VEGF-C and VEGF-D- expressing tumors induce lymphangiogenesis and increase metastasis [10-12]. The coordinate growth of large blood and lymphatic vessel triads thus not only supports tumor growth and fluid drainage, but also likely promotes lymphatic metastasis to draining lymph nodes.

The large blood vessels in the vicinity of B16-F10 melanomas were always functional in circulating blood, however lymphatic vessels were sometimes blocked. Blocked lymph drainage was readily identified upon necropsy after Evans Blue subcutaneous injection into the tail base, with dye usually reaching the ING LN but not the second tier draining AX LN, and this blockade could be confirmed by subcutaneous injection of Texas Red Dextran followed by immunostaining analysis. Our identification of altered lymphatic drainage is relevant for lymphography mapping of the sentinel tumor-draining LN in human cancer patients, for pathology analysis to diagnose whether the tumor has metastasized $[52,53]$. The accuracy of sentinel LN identification has been improved $[54,55]$, however the difficulties in correctly identifying the tumor-draining LN could be caused by blocked lymphatic drainage as experimentally demonstrated here.

Recent studies have identified effects of large LN metastases to block tumor lymphatic drainage in mouse tumor models [56,57]. In our study, mice showing blocked lymph drainage did not yet contain metastases in the ING or AX LNs, even though melanotic metastases consisting of less than 1,000 cells can readily be visualized on the LN cortical surface [23]. Those tumors demonstrating blocked lymph drainage were not larger or located closer to the SE or IF vessel triads relative to tumors with functional lymphatics, so that blocked drainage is not readily explained by tumor overgrowth. However, these tumors could potentially compress the lymphatic vessels to impair lymph drainage. Alternatively, in-transit metastases in tumor-draining lymphatic vessels have been identified in murine and human melanomas [58,59], which could potentially block lymph drainage before lymph node metastases are detectible.

Both of the murine tumor models investigated here exhibited coordinate growth of tumor-draining lymphatic and blood vessel triads, suggesting that tumors produce factors promoting growth of both types of vessels. Members of the VEGF family of endothelial growth factors (VEGF-A, -C, -D) show overlapping specificity for VEGF receptors expressed on lymphatic and blood vessels $[16,60]$, which could potentially drive the concerted growth of both types of vessels $[19,61,62]$. Our RT-PCR analysis of B16-F10 melanoma cells identified abundant VEGF-A, VEGF-C, and VEGF-D expression at the mRNA level (data not shown), and B16-F10 melanomas [63] and DMBA-TPA-induced papillomas [64] produce high levels of VEGF-A protein. It remains to be determined whether these VEGFs or other endothelial growth factors coordinate growth of the blood and lymphatic vessel triads. Investigation of the mechanisms coordinating growth of these peritumoral vessel triads could help to define therapeutic strategies to effectively block both tumor angiogenesis and lymphangiogenesis.

\section{Abbreviations}

AX: Axillary; DMBA/TPA: 7,12-dimethylbenz(a)anthracene/ 12-O-tetradecanoyl-phorbol-13-acetate; IF: Inguinofemoral; ING: Inguinal; LN: Lymph node; RT-PCR: Reverse transcription-polymerase chain reaction; SE: Superficial epigastric; VEGF: Vascular endothelial growth factor.

\section{Competing interests}

The authors declare that they do not have any competing interests.

\section{Author's contributions}

$A R, A C, K K$, and MF conceived and designed the experiments. AR, AC, KK, and MF performed experiments. AR and CK wrote the manuscript. All authors read and approved the final manuscript.

\section{Acknowledgements}

We thank Maribel Harrell, Sheila Ganti, Kay Gurley, and Kendra Byrd for their advice and assistance. This work was supported by National Institutes of Health Grant NCI R01-CA68328 (AR), and NCI Mouse Models of Human Cancer Consortium U01 CA141550 and NIEHS grant RO1 ES020116 (CJK). 


\section{Author details}

${ }^{1}$ Fred Hutchinson Cancer Research Center, Seattle, WA, USA. ${ }^{2}$ Department of Comparative Medicine, University of Washington School of Medicine, 1959 NE Pacific St., Box 357190, Seattle, WA 98195, USA. ${ }^{3}$ Department of Pathology, University of Washington School of Medicine, Seattle, WA, USA.

Received: 3 December 2013 Accepted: 16 May 2014

Published: 21 May 2014

\section{References}

1. Gray H: Anatomy of the human body. Philadelphia: Lea and Febiger; 1918.

2. Sabin FR: The lymphatic system in human embryos, with a consideration of the morphology of the system as a whole. Am J Anat 1909, 9:43-91.

3. Kume T: Specification of arterial, venous, and lymphatic endothelial cells during embryonic development. Histol Histopathol 2010, 25:637-646.

4. Adams RH, Eichmann A: Axon guidance molecules in vascular patterning. Cold Spring Harb Perspect Biol 2010, 2:a001875.

5. Adams RH, Alitalo K: Molecular regulation of angiogenesis and lymphangiogenesis. Nat Rev Mol Cell Biol 2007, 8:464-478.

6. Carmeliet P, Jain RK: Angiogenesis in cancer and other diseases. Nature 2000, 407:249-257.

7. Folkman J, Ingber D: Inhibition of angiogenesis. Semin Cancer Biol 1992 3:89-96.

8. Folkman J: The role of angiogenesis in tumor growth. Semin Cancer Biol 1992, 3:65-71.

9. Nagy JA, Chang SH, Dvorak AM, Dvorak HF: Why are tumour blood vessels abnormal and why is it important to know? Br J Cancer 2009, 100:865-869.

10. Mandriota SJ, Jussila L, Jeltsch M, Compagni A, Baetens D, Prevo R, Banerji S, Huarte J, Montesano R, Jackson DG, Orci L, Alitalo K, Christofori G, Pepper MS: Vascular endothelial growth factor-C-mediated lymphangiogenesis promotes tumour metastasis. EMBO J 2001, 20:672-682

11. Skobe M, Hawighorst $T$, Jackson DG, Prevo R, Janes $L$, Velasco P, Riccardi $L$, Alitalo K, Claffey K, Detmar M: Induction of tumor lymphangiogenesis by VEGF-C promotes breast cancer metastasis. Nat Med 2001, 7:192-198.

12. Stacker SA, Caesar C, Baldwin ME, Thornton GE, Williams RA, Prevo R, Jackson DG, Nishikawa S, Kubo H, Achen MG: VEGF-D promotes the metastatic spread of tumor cells via the lymphatics. Nat Med 2001, 7:186-191.

13. Wiig H, Swartz MA: Interstitial fluid and lymph formation and transport: physiological regulation and roles in inflammation and cancer. Physiol Rev 2012, 92:1005-1060.

14. Hagendoorn J, Tong R, Fukumura D, Lin Q, Lobo J, Padera TP, Xu L, Kucherlapati R, Jain RK: Onset of abnormal blood and lymphatic vessel function and interstitial hypertension in early stages of carcinogenesis. Cancer Res 2006, 66:3360-3364.

15. Cao Y: Emerging mechanisms of tumour lymphangiogenesis and lymphatic metastasis. Nat Rev Cancer 2005, 5:735-743.

16. Shayan $R$, Achen MG, Stacker SA: Lymphatic vessels in cancer metastasis: bridging the gaps. Carcinogenesis 2006, 27:1729-1738.

17. Dvorak HF, Nagy JA, Dvorak JT, Dvorak AM: Identification and characterization of the blood vessels of solid tumors that are leaky to circulating macromolecules. Am J Pathol 1988, 133:95-109.

18. Hilmas DE, Gillette EL: Morphometric analyses of the microvasculature of tumors during growth and after x-irradiation. Cancer 1974, 33:103-110.

19. Scavelli C, Vacca A, Di Pietro G, Dammacco F, Ribatti D: Crosstalk between angiogenesis and lymphangiogenesis in tumor progression. Leukemia 2004, 18:1054-1058

20. Hashizume H, Baluk P, Morikawa S, McLean JW, Thurston G, Roberge S, Jain RK, McDonald DM: Openings between defective endothelial cells explain tumor vessel leakiness. Am J Pathol 2000, 156:1363-1380.

21. Padera TP, Kadambi A, di Tomaso E, Carreira CM, Brown EB, Boucher Y, Choi NC, Mathisen D, Wain J, Mark EJ, Munn LL, Jain RK: Lymphatic metastasis in the absence of functional intratumor lymphatics. Science 2002, 296:1883-1886

22. Fidler IJ: Selection of successive tumor lines for metastasis. Nat New Biol 1973, 242:148-149.

23. Ruddell A, Harrell MI, Furuya M, Kirschbaum SB, Iritani BM: B lymphocytes promote lymphogenous metastasis of lymphoma and melanoma. Neoplasia 2011, 13:748-757.

24. Harrell MI, Iritani BM, Ruddell A: Tumor-induced sentinel lymph node lymphangiogenesis and increased lymph flow precede melanoma metastasis. Am J Pathol 2007, 170:774-786.
25. Giavazzi R, Garofalo A: B16 Melanoma Metastasis. In Methods in Molecular Medicine. Volume 58. 1st edition. Edited by Brooks SA, Schumacher U. Totowa, New Jersey: Humana Press, Inc; 2001:223-229.

26. Quintanilla M, Brown K, Ramsden M, Balmain A: Carcinogen-specific mutation and amplification of Ha-ras during mouse skin carcinogenesis. Nature 1986, 322:78-80.

27. Fidler IJ: Biological behavior of malignant melanoma cells correlated to their survival in vivo. Cancer Res 1975, 35:218-224.

28. Kelly-Spratt KS, Gurley KE, Yasui Y, Kemp CJ: p19Arf supports growth, malignant conversion, and metastasis of Ha-ras driven squamous cel carcinomas through p53 dependent and independent pathways. PLoS Biol 2004, 2:1138-1149.

29. Kemp CJ, Donehower LA, Bradley A, Balmain A: Reduction of p53 gene dosage does not increase initiation or promotion but enhances malignant progression of chemically induced skin tumors. Cell 1993 4:813-822.

30. Farr A, Nelson A, Hosier S, Kim A: A novel cytokine-responsive cell surface glycoprotein defines a subset of medullary thymic epithelium in situ. $\mathrm{J}$ Immunol 1993, 150:1160-1171.

31. Tilney NL: Patterns of lymphatic drainage in the adult laboratory rat J Anat 1971, 109:369-383.

32. Harrell MI, Iritani BM, Ruddell A: Lymph node mapping in the mouse. J Immunol Methods 2008, 332:170-174.

33. Ruddell A, Harrell MI, Minoshima S, Maravilla KR, Iritani BM, White SW, Partridge SC: Dynamic contrast-enhanced magnetic resonance imaging of tumor-induced lymph flow. Neoplasia 2008, 10:706-713.

34. Baluk P, Fuxe J, Hashizume H, Romano T, Lashnits E, Butz S, Vestweber D, Corada M, Molendini C, Dejana E, McDonald DM: Functionally specialized junctions between endothelial cells of lymphatic vessels. J Exp Med 2007, 204:2349-2362.

35. Furuya M, Kirschbaum SB, Paulovich A, Pauli BU, Zhang H, Alexander JS, Farr AG, Ruddell A: Lymphatic endothelial murine chloride channel calcium-activated 1 is a ligand for leukocyte LFA-1 and Mac-1. $\mathrm{J}$ Immunol 2010, 185:5769-5777.

36. Ruddell A, Mezquita P, Brandvold KA, Farr A, Iritani BM: B lymphocyte-specific c-Myc expression stimulates early and functional expansion of the vasculature and lymphatics during lymphomagenesis. Am J Pathol 2003, 163:2233-2245.

37. Makinen T, Adams RH, Bailey J, Lu Q, Ziemiecki A, Alitalo K, Klein R, Wilkinson GA: PDZ interaction site in ephrinB2 is required for the remodeling of lymphatic vasculature. Genes Dev 2005, 19:397-410.

38. van Amerongen MJ, Molema G, Plantinga J, Moorlag H, van Luyn MJ: Neovascularization and vascular markers in a foreign body reaction to subcutaneously implanted degradable biomaterial in mice. Angiogenesis 2002, 5:173-180.

39. Wigle JT, Harvey N, Detmar M, Lagutina I, Grosveld G, Gunn MD, Jackson DG, Oliver G: An essential role for Prox1 in the induction of the lymphatic endothelial cell phenotype. EMBO J 2002, 21:1505-1513.

40. Hallmann R, Mayer DN, Berg EL, Broermann R, Butcher EC: Novel mouse endothelial cell surface marker is suppressed during differentiation of the blood brain barrier. Dev Dyn 1995, 202:325-332.

41. Norrmen C, Ivanov Kl, Cheng J, Zangger N, Delorenzi M, Jaquet M, Miura N, Puolakkainen P, Horsley V, Hu J, Augustin HG, Yla-Herttuala S, Alitalo K, Petrova TV: FOXC2 controls formation and maturation of lymphatic collecting vessels through cooperation with NFATc1. J Cell Biol 2009, 185:439-457.

42. Alitalo K: The lymphatic vasculature in disease. Nat Med 2011, 17:1371-1380

43. Rafii S, Lyden D, Benezra R, Hattori K, Heissig B: Vascular and haematopoietic stem cells: novel targets for anti-angiogenesis therapy? Nat Rev Cancer 2002, 2:826-835.

44. Zumsteg A, Baeriswyl V, Imaizumi N, Schwendener R, Ruegg C, Christofori G: Myeloid cells contribute to tumor lymphangiogenesis. PLoS One 2009, 4:e7067.

45. Karnezis T, Shayan R, Caesar C, Roufail S, Harris NC, Ardipradja K, Zhang YF, Williams SP, Farnsworth RH, Chai MG, Rupasinghe TW, Tull DL, Baldwin ME, Sloan EK, Fox SB, Achen MG, Stacker SA: VEGF-D promotes tumor metastasis by regulating prostaglandins produced by the collecting lymphatic endothelium. Cancer Cell 2012, 21:181-195.

46. Risau W: Mechanisms of angiogenesis. Nature 1997, 386:671-674.

47. Saharinen P, Tammela T, Karkkainen MJ, Alitalo K: Lymphatic vasculature: development, molecular regulation and role in tumor metastasis and inflammation. Trends Immunol 2004, 25:387-395. 
48. Cleaver O, Melton DA: Endothelial signaling during development. Nat Med 2003, 9:661-668.

49. Oliver G: Lymphatic vasculature development. Nat Rev Immunol 2004, 4:35-45.

50. Harvey NL, Oliver G: Choose your fate: artery, vein or lymphatic vessel? Curr Opin Genet Dev 2004, 14:499-505.

51. Simons M, Eichmann A: Physiology. Lymphatics are in my veins. Science 2013, 341:622-624.

52. Morton DL, Wen DR, Wong JH, Economou JS, Cagle LA, Storm FK, Foshag $\sqcup$, Cochran AJ: Technical details of intraoperative lymphatic mapping for early stage melanoma. Arch Surg 1992, 127:392-399.

53. Cheng G, Kurita S, Torigian DA, Alavi A: Current status of sentinel lymph-node biopsy in patients with breast cancer. Eur J Nucl Med Mol Imaging 2011, 38:562-575.

54. Spillane AJ, Brennan ME: Accuracy of sentinel lymph node biopsy in large and multifocal/multicentric breast carcinoma-a systematic review. Eur J Surg Oncol 2011, 37:371-385.

55. Testori A, De Salvo GL, Montesco MC, Trifiro G, Mocellin S, Landi G, Macripo G, Carcoforo P, Ricotti G, Giudice G, Picciotto F, Donner D, Di Filippo F, Soteldo J, Casara D, Schiavon M, Vecchiato A, Pasquali S, Baldini F, Mazzarol $G$, Rossi CR: Clinical considerations on sentinel node biopsy in melanoma from an Italian multicentric study on 1,313 patients (SOLISM-IMI). Ann Surg Oncol 2009, 16:2018-2027.

56. Kwon S, Sevick-Muraca EM: Functional lymphatic imaging in tumor-bearing mice. J Immunol Methods 2010, 360:167-172.

57. Proulx ST, Luciani P, Christiansen A, Karaman S, Blum KS, Rinderknecht M, Leroux JC, Detmar M: Use of a PEG-conjugated bright near-infrared dye for functional imaging of rerouting of tumor lymphatic drainage after sentinel lymph node metastasis. Biomaterials 2013, 34:5128-5137.

58. Scoggins $C R$, Martin RC, Ross Ml, Edwards MJ, Reintgen DS, Urist MM, Gershenwald JE, Sussman JJ, Dirk Noyes R, Goydos JS, Beitsch PD, Ariyan S, Stromberg AJ, Hagendoorn LJ, McMasters KM: Factors associated with false-negative sentinel lymph node biopsy in melanoma patients. Ann Surg Oncol 2010, 17:709-717.

59. Tammela T, Saaristo A, Holopainen T, Yla-Herttuala S, Andersson LC, Virolainen S, Immonen I, Alitalo K: Photodynamic ablation of lymphatic vessels and intralymphatic cancer cells prevents metastasis. Sci Trans/ Med 2011, 3:69ra11.

60. Lohela M, Bry M, Tammela T, Alitalo K: VEGFs and receptors involved in angiogenesis versus lymphangiogenesis. Curr Opin Cell Biol 2009, 21:154-165.

61. Baldwin ME, Stacker SA, Achen MG: Molecular control of Lymphangiogenesis. Bioessays 2002, 24:1030-1040.

62. Nagy JA, Vasile E, Feng D, Sundberg C, Brown LF, Detmar MJ, Lawitts JA, Benjamin L, Tan X, Manseau EJ, Dvorak AM, Dvorak HF: Vascular permeability factor/vascular endothelial growth factor induces Lymphangiogenesis as well as Angiogenesis. J Exp Med 2002, 196:1497-1506.

63. Guba M, von Breitenbuch $P$, Steinbauer M, Koehl G, Flegel S, Hornung M, Bruns CJ, Zuelke C, Farkas S, Anthuber M, Jauch KW, Geissler EK: Rapamycin inhibits primary and metastatic tumor growth by antiangiogenesis: involvement of vascular endothelial growth factor. Nat Med 2002, 8:128-135.

64. Ruddell A, Kelly-Spratt KS, Furuya M, Parghi SS, Kemp CJ: p19/Arf and p53 suppress sentinel lymph node lymphangiogenesis and carcinoma metastasis. Oncogene 2008, 27:3145-3155.

doi:10.1186/1471-2407-14-354

Cite this article as: Ruddell et al:: Tumors induce coordinate growth of artery, vein, and lymphatic vessel triads. BMC Cancer 2014 14:354.

\section{Submit your next manuscript to BioMed Central and take full advantage of:}

- Convenient online submission

- Thorough peer review

- No space constraints or color figure charges

- Immediate publication on acceptance

- Inclusion in PubMed, CAS, Scopus and Google Scholar

- Research which is freely available for redistribution

Submit your manuscript at www.biomedcentral.com/submit
Ciomed Central 